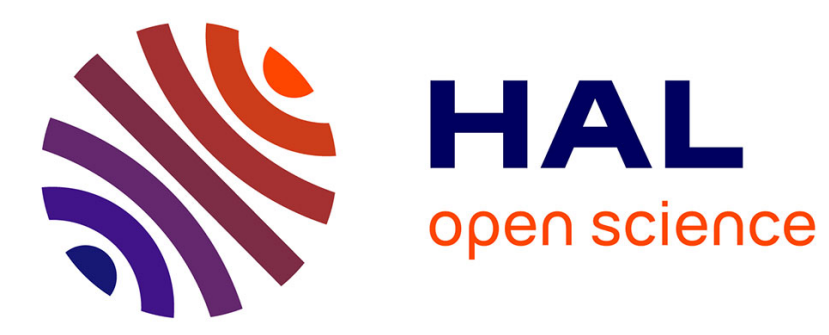

\title{
Neutron scattering at high pressure
}

\author{
D.B. Mcwhan
}

\section{To cite this version:}

D.B. Mcwhan. Neutron scattering at high pressure. Revue de Physique Appliquée, 1984, 19 (9), pp.715-718. 10.1051/rphysap:01984001909071500 . jpa-00245243

\section{HAL Id: jpa-00245243 https://hal.science/jpa-00245243}

Submitted on 1 Jan 1984

HAL is a multi-disciplinary open access archive for the deposit and dissemination of scientific research documents, whether they are published or not. The documents may come from teaching and research institutions in France or abroad, or from public or private research centers.
L'archive ouverte pluridisciplinaire HAL, est destinée au dépôt et à la diffusion de documents scientifiques de niveau recherche, publiés ou non, émanant des établissements d'enseignement et de recherche français ou étrangers, des laboratoires publics ou privés. 


\title{
Neutron scattering at high pressure
}

\author{
D. B. McWhan \\ Room 1D-234, Murray Hill, New Jersey 07974, Ü.S.A.
}

\begin{abstract}
Résumé. - Nous présentons les différentes techniques utilisées lors d'expériences de diffusion élastique et inélastique de neutrons réalisées sur des sources conventionnelles (réacteurs) ou pulsées - les enceintes de pression dont on dispose dans le plupart des centres de recherche neutronique permettent d'atteindre $5 \mathrm{GPa}(50 \mathrm{kbar})$; des tentatives pour atteindre $10 \mathrm{GPa}$ ont été effectuées. Dans le cas de diffusion élastique, sous haute pression, on compare les résultats expérimentaux obtenus par diffusion des neutrons et ceux obtenus par diffusion des R.X. en utilisant une source à anode tournante ou le rayonnement synchrotron.
\end{abstract}

Abstract. - The techniques to do elastic and inelastic neutron scattering at steady-state and pulsed sources are
reviewed. The pressure cells available at most neutron scattering centres are capable of reaching pressures of the
order of $5 \mathrm{GPa}(50 \mathrm{kbar})$, and attempts to reach $10 \mathrm{GPa}$ have been made. For elastic scattering, a comparison is
made between neutron scattering and X-ray scattering at high pressure using rotating anode or synchrotron sources.

An important use of the pressure variable is to understand phenomena observed at atmospheric pressure. Often, it is possible to tune competing interactions by changing the pressure and to test models which have been proposed for different types of phase transitions. Neutron and X-ray scattering measurements have been used to study a wide variety of properties from phase transitions to lattice dynamics, and it is the purpose of this paper to compare these two scattering probes and to illustrate the types of problems which have been studied. X-ray scattering at high pressure is the subject of two other papers at this workshop [1, 2], and the techniques to do neutron scattering at high pressure are summarized below. The advantages and disadvantages of each probe are discussed, and a summary of recent studies is presented.

\section{Experimental techniques.}

Apparatus for making neutron scattering measurements at high pressure falls, more or less, into four categories; and those have been discussed extensively in the literature [3-5]. For measurements under hydrostatic or quasihydrostatic pressure there are three different ranges of pressure, and the maximum attainable pressure in each range is governed by materials considerations. Up to pressures of the order of $1 \mathrm{GPa}(1 \mathrm{GPa}=10 \mathrm{kbar})$ unsupported pressure cells can be made of high strength aluminum alloys. As the absorption cross section of aluminum to thermal neutrons is quite low, there is typically less than
$10 \%$ attenuation of the incident and scattered neutrons. Sample volumes of the order of $1.5 \mathrm{~cm}^{3}$ are available and helium gas is used as the pressure transmitting medium. For special purposes such as ultralow temperatures or high magnetic fields, it is often more convenient to use a clamp device in which the sample is mounted in a small cylinder containing a fluid and the cylinder is, in turn, mounted in a piston cylinder cell made of beryllium copper [6,7]. The pressure is raised to the desired level at room temperature and then the pressure is clamped by a locking nut. This clamp device, which is typically $2.5 \mathrm{~cm}$ in diameter and $8-12 \mathrm{~cm}$ long, is then mounted in a dilution refrigerator or in a superconducting magnet. In these clamp devices the sample volume is only of the order of $3 / 4 \mathrm{~cm}^{3}$ or less. There is the further problem that the pressure transmitting medium, which is usually a fluorocarbon on a deuterated hydrocarbon or alcohol, freezes on cooling producing a small uniaxial component in addition to the hydrostatic pressure. In some cases this can lead to qualitative changes in the physics and therefore the effect of uniaxial stress has to be assessed in each experiment [8]. There is also a change in pressure on cooling resulting from differential thermal expansion among the components of the apparatus and the sample. The properties measured as a function of temperature do not follow a true isobar, and a pressure correction has to be made [7]. The attenuation of the neutron beam is of the order of $50 \%$ in these cells. 
In order to achieve pressures above $10 \mathrm{GPa}$ it is necessary to use a stronger material which is still relatively transparent to neutrons, and almost all existing equipment has used high density polycrystalline $\mathrm{Al}_{2} \mathrm{O}_{3}$ for the pressure cell. (A typical cell is shown in Fig. 1). In addition, the $\mathrm{Al}_{2} \mathrm{O}_{3}$ needs to be externally supported, thereby restricting the window.

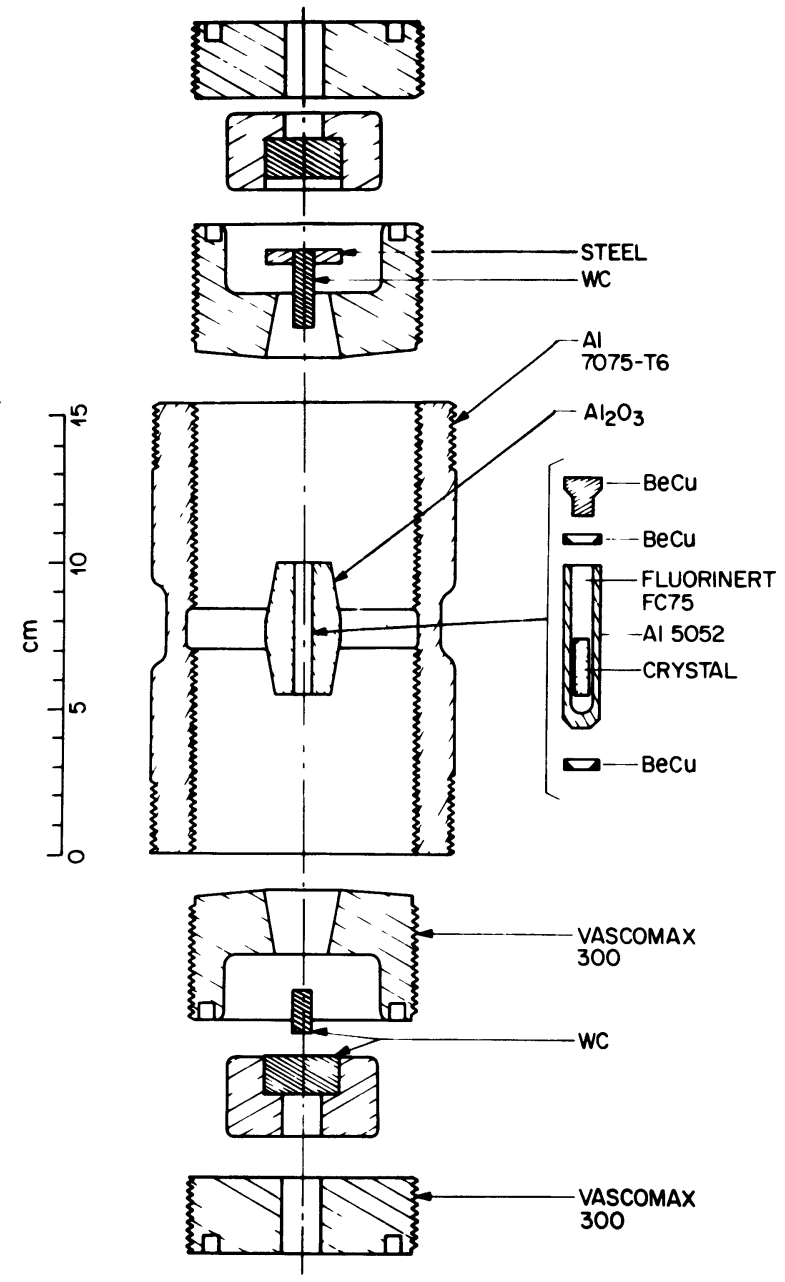

Fig. 1. - Exploded view of a typical clamp device for neutron scattering at pressures of $4.5 \mathrm{GPa}$ and low temperatures.

In time of flight measurements only a few windows at fixed angles are needed for the incident and diffracted neutron beams to study polycrystalline materials. For studies of single crystals using either time of flight or triple axis spectrometers a cylindrical window between the support blocks is needed to cover a plane in reciprocal space. The restricted height of the window further reduces the available sample volume to less than $0.2 \mathrm{~cm}^{3}$, and if a single crystal is to be mounted so as to avoid contact with the walls of the pressure cell at the highest pressures, then a typical sample might be only $0.1 \mathrm{~cm}^{3}$. The maximum pressure attainable in piston cylinder devices is 4-5 GPa, and this is determined by the pressure at which the unsupported tungsten carbide piston fails.
In order to achieve pressures above $5 \mathrm{GPa}$ it is necessary to change from a piston cylinder design to an anvil apparatus. Opposed diamond anvil high pressure apparatus for X-ray studies has achieved quasihydrostatic pressures above $100 \mathrm{GPa}(1 \mathrm{mega}-$ bar), but with samples of the order of $3 \times 10^{-7} \mathrm{~cm}^{3}$. Quasihydrostatic pressures of $10 \mathrm{GPa}$ were achieved in an exploratory study using Bridgman anvils made of $\mathrm{Al}_{2} \mathrm{O}_{3}$ with hardened steel support rings [3]. The sample volume was $0.05 \mathrm{~cm}^{3}$ and a time of flight geometry was used with the incident neutron beam normal to the disc shaped sample and the diffracted beam at $90^{\circ}$. This single study was made about 15 years ago, and one might speculate that higher pressures could be achieved with large sintered diamond anvils. However, to date, all of the published neutrons scattering results at high pressure have been made below $5 \mathrm{GPa}$ in supported piston cylinder devices using $\mathrm{Al}_{2} \mathrm{O}_{3}$ or below $1 \mathrm{GPa}$ in unsupported cells.

The fourth type of apparatus is that for measurements under uniaxial stress. The maximum stresses which can be achieved depend mainly on the strength of the material being studied and on alignment of the apparatus. Typically, for semiconductors, stresses of the order of $2 \mathrm{GPa}$ have been achieved, and details of the design of apparatus for neutron scattering measurements under uniaxial stress are found in references [9] and [10].

\section{Comparison of $\mathrm{X}$-ray and neutron scattering at high pressure.}

The major advantage of neutron scattering is the ability to do inelastic scattering and to probe elementary excitations, but for elastic scattering, the choice of scattering probe varies from problem to problem. The difference in sample volume of $10^{6}$ between high pressure piston cylinder devices for neutron scattering and diamond anvil devices for $\mathrm{X}$-ray scattering goes a long way toward offsetting the much larger fluxes of X-ray sources over neutron sources. In addition, neutrons have the added advantages of 1) low absorption cross sections, 2) atomic scattering factors which are constant, and 3) well characterized and easily modelled resolution functions. The first is very important in uniaxial stress experiments where one wants to probe the centre of the sample where the stress distribution is more homogeneous. The second and third points are important for crystallographic studies. However, in problems involving subtle splittings of reflections, the high resolution which can be obtained using a synchrotron source and perfect crystal monochromator and analyser are superior to neutron scattering. These different points are illustrated in figure 2 where longitudinal scans through the (10.0) of $2 \mathrm{H}-\mathrm{TaSe}_{2}$ are compared for two X-ray sources and a reactor source. For the X-ray measurements a crystal of $2 \mathrm{H}-\mathrm{TaSe}_{2}$ was mounted in a Mer- 


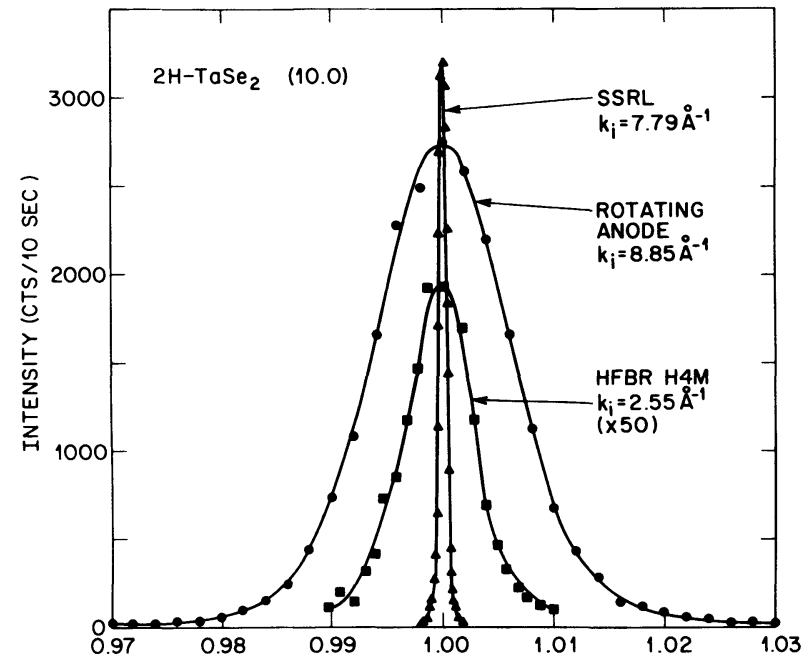

Fig. 2. - Comparison of (10.0) reflection of $2 \mathrm{H}-\mathrm{TaSe}_{2}$ in a diamond anvil cell observed using a rotating anode or synchrotron source and in the cell shown in figure 1 using a reactor source as described in the text.

ril-Bassett diamond anvil cell using the standard 4: 1 mixture of methanol-ethanol as the pressure transmitting medium. The Rigaku rotating anode source operating at $8 \mathrm{~kW}$ used a molybdenum target and the $0.2 \times 2 \mathrm{~mm}$ high brilliance filament. A vertically bent pyrolytic graphite monochromator (00.4) was used in a symmetric configuration to re-image the source on the sample. A slit before the sample and a flat lithium fluoride analyser were used to maximize the resolution [11]. The same sample and diamond cell were used at the Stanford Synchrotron Radiation Laboratory, together with a double crystal Si(111) monochromator and with a $\mathrm{Ge}(111)$ analyser [12]. The third curve was obtained at the High Flux Beam Reactor at Brookhaven National Laboratory using vertically bent graphite (002) monochromator and (004) analyser in conjunction with $20-40^{2}-10$ collimation and a graphite filter. A $0.06 \mathrm{~cm}^{3}$ sample was mounted in an aluminum cell containing Fluorinert as the pressure transmitting medium, and this cell was loaded into the supported $\mathrm{Al}_{2} \mathrm{O}_{3}$ pressure apparatus to give a (h0.1) scattering plane [13]. In order to resolve the subtle splitting of the charge density wave superlattice reflections at low temperatures, it is necessary to be able to see a splitting of 0.003 . The rotating anode data cannot resolve this splitting, and it is poorly resolved in the neutron scattering data [13]. Clearly, following the pressure dependence of a phase transition of this subtlety could be done with more accuracy on a synchrotron source rather than a reactor source.

The real power of elastic neutron scattering at high pressure for polycrystalline samples lies in the use of profile refinement techniques. By careful collimation in a time of flight measurement, only neutrons scattered from the sample are observed, and contamination coming from the pressure cell itself can be virtually eliminated. A number of structural refinements have been done at Argonne both on the steady state source and, more recently, on the pulsed source [14].

For inelastic scattering measurements the background is usually only about $50 \%$ higher than in a typical low temperature measurement on a triple axis spectrometer because $\mathrm{Al}_{2} \mathrm{O}_{3}$ is very incompressible, and the dispersion relations for the acoustic phonon modes have correspondingly large slopes. The relatively small sample volume makes it difficult to measure excitations with little dispersion that have energies much greater than $10 \mathrm{meV}$, but excitations as high as $36 \mathrm{meV}$ have been observed [15].

\section{Phase transitions.}

The majority of the neutron scattering studies at high pressure have concerned different types of phase transitions. The temperature-pressure phase diagrams of a number of systems with incommensurate-commensurate phase transitions have been studied. These include the charge density wave transitions in $2 \mathrm{H}$ $\mathrm{TaSe}_{2}[11,13]$, the spin density wave transitions in $\mathrm{Cr}$ alloys [7], the $2 k_{\mathrm{F}}$ and $4 k_{\mathrm{F}}$ transitions in the quasione-dimensional magnetic conductor MEM$(\mathrm{TCNQ})_{2}$ [16], and thiourea [17]. Thiourea is a particularly interesting example in that it demonstrates the advantage of combining pressure measurements with those of other variables such as temperature and electric field. At low temperatures the stable phase has a modulation wave vector of $\delta=1 / 9$ and the application of a small electric field leads to a transition to a phase with $\delta=1 / 8$. At high pressure a phase transition to a phase with $\delta=1 / 7$ is observed [17]. Recent X-ray measurements at higher electric fields revealed a series of phases with relatively high commensurability order [18]. These new phases could be understood by combining the results of the low electric field and the high pressure measurements. With increasing field there is a shift in the sequences of antiphase domains with an increase in the fraction of the domains corresponding to that observed at high pressure $(\delta=1 / 7)$ with respect to the fraction of those observed at low fields $(\delta=1 / 8)$. The sequence of high commensurability phases was accurately given by $\bar{q}=(n+1) /(7 n+8)$ with $n=1,3$ and 5 . This reflects the stepwise progression with increasing electric field toward the phase found at high pressure.

Another area of recent interest has been pressure induced staging transitions in alkali graphite intercalation compounds. An increase in stage from stage 2 to stage 3 accompanied by an increase in in-plane density was observed in $\mathrm{KC}_{24}$ [19]. The first example of a fractional stage $3 / 2$ in $\mathrm{KC}_{8}$ was observed by neutron scattering [20] and both of these results are in good agreement with a recent theoretical pressurecomposition phase diagram [21].

The full power of neutron scattering was brought to bear on the isostructural transition to the mixed 
valence state in $\mathrm{SmS}$. The local atomic structure was probed by studying the disappearance of the $\mathrm{Sm}^{2+}$ ${ }^{7} F_{0} \rightarrow{ }^{7} F_{1}$ excitation at the transition [15]. The magnetic form factor of a well defined $\mathrm{Sm}^{3+}$ ion is unusual in that there is a maximum at a finite value of $\sin \theta / \lambda$, but this was not observed in the mixed valence phase above $P=0.6 \mathrm{GPa}^{6}$. Finally, a softening near the zone centre and a stiffening near the zone boundary of the longitudinal acoustic phonon mode was observed in the high pressure phase [22].
A number of second order phase transition which are accompanied by a softening of an acoustic phonon mode $\left(\mathrm{TeO}_{2}^{23}, \mathrm{NiF}_{2}^{14}\right)$ or of a magnetic exciton $\left(\mathrm{PrSb}^{24}, \mathrm{Pr}^{25}\right)$ have been found, and the pressure dependence of the modes studied in detail. This brief summary illustrates that neutron scattering at pressures up to $4 \mathrm{GPa}$ and at temperatures between $T=4.2$ and $300 \mathrm{~K}$ has made important contributions to our understanding of a wide variety of phase transitions.

\section{References}

[1] Holzapfel, W., Revue Phys. Appl., this issue.

[2] Buras, B., Ibid, Ref. 1.

[3] Brugger, R. M., Bennion, R. B., Worlton, T. G. and Myers, W. R., Trans. Amer. Cryst. Soc. 5 (1969) 141.

[4] McWhan, D. B., in High Pressure Science and Techno$\log y, \mathrm{~K}$. D. Timmerhaus and M. S. Barber, eds. (Plenum, N.Y.) 1979, p. 292.

[5] Vettier, C., in Neutron Scattering-1981, J. Faber, Jr., ed., AIP Conf. Proc. 89 (1982) 121.

[6] Moon, R. M., Koehler, W. C., McWhan, D. B. and Holtzberg, F., J. Appl. Phys. 49 (1978) 2107.

[7] Mizuki, J. and EndoH, Y., J. Phys. Soc. Jpn 50 (1981) 914.

[8] McWhan, D. B., in High Pressure in Research and Industry, C. M. Backman, T. Johannisson and L. Tegner, eds., (Ankitektropia, Uppsala) 1981. p. 612.

[9] Draperi, A., Herrmann-Ronzaud, D. and Paureau, J., J. Phys. E 9 (1976) 174.

[10] Pretchel, J., Luscher, E. and Kalus, J., J. Phys. E 10 (1977) 432.

[11] McWhan, D. B., Fleming, R. M., Moncton, D. E. and Di Salvo, F. J., Phys. Rev. Lett. 45 (1980) 269.

[12] McWhan, D. B., Fleming, R. M. and Moncton, D. E., unpublished results.

[13] McWhan, D. B., Axe, J. D. and Youngblood, R., Phys. Rev. B 24 (1981) 5391.
[14] For example, see : Jorgensen, J. D., Worlton, T. G. and Jamieson, J. C., Phys. Rev. B 17 (1978) 2212.

[15] McWhan, D. B., Shapiro, S. M., Eckert, J., Mook, H. A. and Birgeneau, R. J., Phys. Rev. B 18 (1978) 3623.

[16] Bloch, D., Voiron, J., Vettier, C., Bray, J. W. and Oostra, S., Physica 119B (1983) 43.

[17] Denoyer, F., Moudden, A. H., Currat, R. Vettier, C., Bellamy, A. and Lambert, M., Phys. Rev. B 25 (1982) 1697.

[18] Moudden, A. H., Moncton, D. E. and Axe, J. D., Phys. Rev. Lett. 51 (1983) 2390.

[19] Clark, R., Wada, N. and Solin, S. A., Phys. Rev. Lett. 44 (1980) 1616.

[20] Fuerst, C. B., Fischer, J. E., Axe, J. D., Hastings, J. B and McWhan, D. B., Phys. Rev. Lett. 50 (1983) 357.

[21] Hawrylak, P. and Subbaswamy, K. B., Phys. Rev. B 28 (1983) 4851.

[22] Mook, H. A., McWhan, D. B., Holtzberg, F., Phys. Rev. B 25 (1982) 4321.

[23] McWhan, D. B., Birgeneau, R. J., Bonner, W. A., TAUB, H. and Axe, J. D., J. Phys. 8 (1975) L81.

[24] MCWhan, D. B., Vettier, C., Youngblood, R. and ShIRANe, G., Phys. Rev. B 20 (1979) 4623.

[25] McEwen, K. A., Stirling, W. G. and Vettier, C., Phys. Rev. Lett. 41 (1978) 343. 